\title{
INFLUENCIA DEL APRENDIZAJE ORGANIZACIONAL EN EL PROCESO DE INNOVACIÓN. UNAAPLICACIÓN EN NUEVAS EMPRESAS DE BASE TECNOLÓGICA
}

Influence of Organizational Learning in the Innovation Process. An Application in New Technology Based Firms

\author{
JULIO CÉSAR ACOSTA PRADO \\ NÉSTOR JUAN SANABRIA LANDAZÁBAL
}

\section{RESUMEN}

En esta investigación se analiza la influencia del aprendizaje organizacional en el proceso de innovación de las Nuevas Empresas de Base Tecnológica (NEBT) del Parque Científico de Madrid (PCM) y del Parque Científico Leganés Tecnológico (LEGATEC), localizadas en la comunidad de Madrid, España. La metodología aplicada es de tipo cualitativa basada en el estudio de casos de siete empresas en los sectores de ingeniería, biotecnología y tecnologías de la información y de las comunicaciones. El resultado obtenido muestra que el aprendizaje organizacional influye de forma positiva en el desarrollo de innovaciones y favorece la gestión eficiente de las empresas.

Palabras clave: Aprendizaje organizacional, innovación, nuevas empresas de base tecnológica (NEBT), investigación y desarrollo (I+D).

\section{ABSTRACT}

In this paper analyzes the organizational learning as process of innovation in New Technology Based Firms (NTBF) of the Science Park of Madrid and of the Science Park Technological Leganes (LEGATEC), localized in the Community of Madrid, Spain. The applied methodology is qualitative based on case studies of seven firms of sectors in information-communication technologies, engineering, and biotechnology. The results show that organizational learning has a positive influence on the development of innovations and helps an efficient management of the firms.

Keyworks: Organizational learning, innovation, new technology-based firms (NTBF), research and development (R\&D).

\section{INTRODUCCIÓN}

El aprendizaje organizacional es uno de los conceptos más amplios y de 
múltiples dimensiones dentro de la literatura de la organización de empresas. Significa esto que un elevado número de autores hacen referencia a él en sus trabajos e investigaciones, pues no existe una única definición ni de lo que es el aprendizaje ni de cómo se desarrolla dentro de la organización (Fiol y Lyles, 1985; Huber, 1991; Weick, 1991; DiBella y Nevis, 1998; Vera y Crossan, 2000). Estos estudios e investigaciones, analizan mayormente el aprendizaje por sus efectos, reconociendo que es una condición para el cambio basado en el estado del conocimiento de una organización. Se trata de una concepción que engloba los componentes cognitivos y de comportamiento vinculados al conocimiento (Argyris y Schön, 1978; Duncan y Weiss, 1979; Garvin, 1993; Senge, 1990; Walsh y Ungson, 1991; Crossan, Lane y White, 1999; Rant, 2001), según la cual el aprendizaje organizacional representa la transformación tanto del modo de pensar las cosas, como del modo de hacer las cosas. Consecuentemente, el resultado inmediato debe ser un conocimiento nuevo o mejorado, siendo el punto central del aprendizaje establecer una experimentación reactiva basada en la prueba y el error y, al mismo tiempo, hacer posible una resolución de problemas con carácter anticipativo.

De todo ello se deduce que todas las organizaciones deben comprometer una parte de sus recursos en el aprendizaje como parte de su desarrollo, pues aquellas organizaciones incapaces de hacer del aprendizaje una cualidad esencial, sencillamente, tienden a desaparecer (Nevis et al., 1995). Lo lógico es considerar que el aprendizaje en la organización constituye un comportamiento sistemático e inmanente a la misma (Duncan y Weiss, 1979). No es una elección, sino algo que forma parte de la definición de las organizaciones como sistemas complejos (Alle, 1997). Así lo han reconocido numerosos autores (Levitt y March, 1988; Leonard Barton, 1993, 1995; DiBella, Nevis y Gould, 1996; Appelbaum y Reichart, 1997; Beveridge, Gear y Minkes, 1997; DiBella y Nevis, 1998; Van der Krogt, 1998, entre otros), para quienes las organizaciones se comportan como sistemas de aprendizaje desarrollados espontáneamente en un determinado contexto. Por tanto, cuanto mayor sea la capacidad de aprendizaje del sistema, 
mayor será la probabilidad de alcanzar y mantener un alto grado de innovación y el éxito en un entorno cambiante.

Recogiendo las argumentaciones previas, en esta investigación se entiende el aprendizaje organizacional como un proceso sistémico dotado de una cartera o estructura de conocimientos que evoluciona constantemente, como respuesta a las transformaciones del entorno que se producen en cada situación concreta (Acosta, 2010). Así pues, es perfectamente posible atribuir al conocimiento las propiedades de un objeto estático que se puede almacenar, manipular, organizar, recuperar, e incluso, medir en cierto grado (Alle, 1997; Zack, 1999) y, al mismo tiempo, el conocimiento es un concepto dinámico que debe ser preferentemente descrito como el proceso de conocer (Polyani, 1962) y, por ende, de actuar dentro de la organización (Nonaka, 1999). Concretamente, el conocimiento es el objeto inicial y resultante del aprendizaje organizacional y, a su vez, de la innovación, por lo que el vínculo existente entre el conocimiento y la acción es posible gracias al aprendizaje (Acosta-Prado, 2010).

En este contexto, el objetivo de esta investigación es analizar la influencia del aprendizaje organizacional en el proceso de innovación de las NEBT del PCM y LEGATEC, localizadas en la Comunidad de Madrid, España.

Teniendo en cuenta el objetivo de investigación y sus fundamentos teóricos, la aplicación de la metodología de investigación es de tipo cualitativa basada en el estudio de siete casos. Las principales aportaciones de este estudio han sido tanto el análisis del aprendizaje organizacional en el proceso de innovación de las NEBT, escasamente estudiadas, como proponer aplicaciones de los resultados obtenidos en la gestión de las empresas.

\section{FUNDAMENTOS TEÓRICOS}

Como se ha señalado, las definiciones acerca del aprendizaje organizacional difieren entre sí en la medida que adoptan un determinado enfoque u otorgan una mayor importancia a unos aspectos sobre otros; así, por 
ejemplo, el aprendizaje organizacional se ha venido relacionando con aspectos tan variados como: las rutinas, el conocimiento, la capacidad o las competencias de la organización para realizar acciones productivas, interpretar y dar sentido al comportamiento organizacional, así como detectar y corregir errores, entre otros.

Estas consideraciones sobre el aprendizaje permiten abordar el estudio de los procesos en virtud de los cuales la organización desarrolla las rutinas y capacidades esenciales, que favorecen la innovación como un fenómeno organizacional complejo construido mediante procesos de aprendizaje internos y externos, cuya importancia no es tanto el conjunto de recursos y capacidades existentes, sino los mecanismos que utilizan las empresas para aprender y acumular nuevas capacidades y habilidades capaces de reconocer las señales del entorno y que se materializan en mejoras y nuevos desarrollos de procesos y de productos (Teece, Pisano y Shuen, 1997).

Un criterio que puede servir para diferenciar los distintos enfoques y las definiciones propuestas del aprendizaje y su influencia en el desarrollo de innovaciones, se concentran en dos enfoques principales. En primer lugar, en el enfoque adaptativo los autores asocian el concepto de aprendizaje organizacional al desarrollo de comportamientos adaptativos de la organización, es decir, se considera el aprendizaje organizacional como un proceso de adaptación al entorno (Cyert y March, 1963; Cangelosi y Dill, 1965; March y Olsen, 1976; Duncan y Weiss, 1979; Hedberg, 1981). En este proceso de ajuste, la organización aprende, siendo el conocimiento y experiencia generados en la interacción con el entorno, la base de su adaptación y actuación al mismo.

En segundo lugar, otros autores abordan el estudio del aprendizaje organizacional y de la innovación desde un enfoque proactivo. Estos lo relacionan con la capacidad de la organización para transformarse y cambiar (Shrivastava, 1983; Swieringa y Wierdsma, 1992; Kim, 1993). También, consideran que la organización que aprende es aquella capaz de provocar su propia trasformación o realidad (Garratt, 1987, 1990, 1992; Bahlmann, 1990; Senge, 1990; Swieringa y Wierdsma, 1992; Landier, 1992; Watkins y 
Marsick, 1993; Redding y Catalanello, 1994). Los autores mencionados no solo consideran a la organización que aprende con capacidad de adaptarse a su entorno, sino también, capaz de promover su propio cambio incidiendo en el entorno.

Un estudio integrador de ambos enfoques, tanto adaptativo como proactivo, es el realizado por Argyris y Schön (1978), que supone la adaptación por influencia del entorno y la acción transformadora de la organización en sí misma, de acuerdo a su realidad.

A este respecto, las contribuciones de Senge (1990) desde su perspectiva sistémica sostienen que la clave para que una organización aprenda radica en su comprensión global y de las interrelaciones entre sus partes componentes. Define el concepto de aprendizaje organizacional como sinónimo de organización inteligente. Además, indica que son los miembros de esta quienes expanden continuamente su aptitud para crear los resultados que desean, donde se cultivan nuevos y expansivos patrones de pensamiento, donde las aspiraciones colectivas quedan en libertad, y donde la gente continuamente aprende a aprender en conjunto.

Un segundo criterio vincula el aprendizaje organizacional con la innovación desde el enfoque de conocimiento. Este agrupa diversas perspectivas. Entre ellas se encuentran los estudios centrados en la problemática de la creación de conocimiento (Dodgson, 1993; Nonaka y Takeuchi, 1995; Sánchez y Heene, 1997; Nonaka e Ichijo, 1997; Nonaka, Reinmoeller y Senoo, 1998) los que analizan y describen la conversión del conocimiento por niveles de aprendizaje, es decir, la integración del conocimiento individual en organizacional (Nonaka y Takeuchi, 1995). Asimismo, Huber (1991), que se centra en la adquisición de conocimiento por parte de la organización. También las aportaciones de Marengo (1991) y Andreu y Ciborra (1994, 1995, 1996), relacionan el aprendizaje al proceso de creación de nuevas competencias o capacidades organizacionales que favorecen el resultado innovador.

A los trabajos de los autores mencionados, se añaden investigaciones 
que sostienen que el aprendizaje organizacional es consecuencia de la ampliación de conocimientos de la organización, debido a la adquisición de conocimiento fecundado en los procesos de resolución de problemas de la misma (Probst y Büchel, 1995; Revilla, 1998).

De acuerdo a las aportaciones recogidas en torno al aprendizaje organizacional se puede decir que siguen una marcada distinción conforme al momento en que fueron desarrollados, desde un enfoque adaptativo, considerado como una vía de adaptación al entorno; desde un enfoque proactivo, entendido el aprendizaje como una clave del desarrollo y transformación organizacional impulsado por la propia organización; o bien desde el enfoque de conocimiento, que concibe las organizaciones como un sistema abierto, y el aprendizaje organizacional como un proceso institucionalizado (el conocimiento individual se convierte en organizacional cuando modifica, crea o reemplaza las rutinas, reglas, procedimientos, etc.). Con base en este conocimiento las organizaciones se construyen y operan con capacidad para responder a la incertidumbre del entorno.

Esta investigación se centra en el enfoque de conocimiento por considerar con se ajusta más al perfil de las NEBT, cuya actividad productiva y comercial se basa fundamentalmente en la explotación de una innovación de producto, que operan en sectores intensivos en conocimiento o de tecnología alta y media-alta, que realizan gasto de I+D y además no tienen más de tres años de antigüedad.

\subsection{Relación aprendizaje organizacional e innovación}

Como ya hemos señalado, cada vez se relaciona más el aprendizaje organizacional y el desarrollo de innovaciones. Desde esta perspectiva se considera que la innovación es un proceso de acumulación y combinación de nuevos conocimientos (Kogut y Zander, 1992), que tienen como resultado la generación de un círculo virtuoso del cual se obtendrán nuevos productos y procesos que reforzarán la obtención de resultados superiores (Acosta, 2010). Por tanto, la innovación es un proceso vinculado a la introducción de cambios en las organizaciones en términos de aprendizaje y conocimiento (Muñoz-Seca y Riverola, 1997). 
Ahora bien, el hecho de analizar el aprendizaje organizacional en el proceso de innovación, engloba un amplio conjunto de actividades dentro de las empresas que contribuyen a generar nuevos conocimientos o mejorar los ya existentes. Estos son aplicados en la obtención de nuevos bienes y servicios y nuevas formas de producción (López, Montes, Vásquez y Prieto, 2004). Todo ello está determinado por la relación entre las características organizacionales y sus resultados y por la identificación y viabilidad del cambio, así como la adecuación de las condiciones, el contexto, o las capacidades que hagan más eficiente y más rápida la producción de innovaciones.

En este contexto, Rogers (1983), a partir del concepto innovación del conocimiento, entiende esta como un proceso informacional en el cual el conocimiento es adquirido, procesado y transferido. Para ello, la organización debe reconocer y aprovechar nuevas oportunidades por medio de la creación y utilización de los conocimientos necesarios para desarrollar capacidades y renovar las existentes (Hamel y Prahalad, 1993).

Por tanto, la innovación es un proceso a través del cual la empresa puede llevar a cabo cambios más profundos y realizar avances científicos y tecnológicos (Benavides, 1998), incorporándolos a aquellos nuevos productos y/o procesos productivos que realiza con el objetivo de adaptarse al entorno y mejorar su posición competitiva (López, et al., 2004).

West y Farr (1990) apuntan que ciertamente toda innovación en términos organizacionales es cambio, aunque no todo cambio es innovación. De este modo, esta constituye una dimensión del cambio organizacional que recoge la intencionalidad del beneficio a obtener, basado en el desarrollo y explotación de conocimientos y aprendizajes determinantes en el resultado innovador (Cohen y Walsh, 2000; Cohen, et al., 2002).

En definitiva, la materialización del aprendizaje organizacional en innovación es el resultado de un proceso prolongado y de acumulación de conocimientos dentro de la empresa que puede verse afectado por la base de conocimientos y aprendizaje facilitadores o inhibidores de dicha capacidad, que implican tanto los efectos de apropiabilidad y la obtención de 
GESTIÓN DEL CONOCIMIENTO Y CAPACIDAD DE INNOVACIÓN

Modelos, Sistemas y Aplicaciones

conocimiento, como la protección de los resultados competitivos (Cohen y Levinthal, 1990).

\subsection{Revisión de estudios empíricos sobre el aprendizaje organizacional como fuente de innovación}

En cuanto al aprendizaje organizacional es importante destacar que una relación muy directa es la que existe entre la base de conocimientos de la organización y el desarrollo de innovaciones (McEvily y Chakravarthy, 2002). Esta situación obedece a que dicho aprendizaje está íntimamente ligado a la capacidad de innovación, no solo porque en él se van a incluir las medidas de input, sino porque además, la capacidad de innovación de una empresa depende, en gran parte, de los conocimientos y la experiencia que posea con anterioridad. Esto será determinante en la acumulación de más activos relacionados con ello y le permitirá incrementar el éxito del nuevo producto (Adner, 2002).

Tradicionalmente en las actividades del proceso de innovación, la I+D es la que más atención ha recibido por parte de la literatura y que el esfuerzo que realiza una empresa se puede representar mediante el gasto de I+D, la intensidad de I+D (relación entre el gasto de I+D y la facturación), la participación en proyectos de I+D con instituciones u organismos de investigación o la existencia formal de un departamento de I+D. Sin embargo, elevados niveles de intensidad en I+D (medida de input frecuentemente utilizada en estudios empíricos) no siempre conllevan resultados innovadores de éxito, mientras que los datos referidos a gastos de I+D sí son un input crítico en el proceso de innovación (Adams, Bessant y Phelps, 2006).

Algunos estudios empíricos muestran que existe un efecto positivo entre el gasto o intensidad en I+D y la innovación de proceso y de producto, siendo esta última la más destacada (Yam, Cheng, Fai y Tang, 2004; Huergo, 2006; Gallego y Casanueva, 2007; Hegde y Shapira, 2007). También encuentran que la gestión de las actividades de I+D tiene una relación positiva con la innovación de proceso (Huergo, 2006), y de producto (Prajogo y Ahmed, 2006), concretamente en la capacidad de la empresa para integrar las estrategias de I+D, implantar los proyectos y gestionarlos (Yam, et al., 2004). 
En relación al grado de novedad, la literatura académica presenta escasos estudios relacionados con el aprendizaje, por lo que no se puede mostrar una influencia positiva entre la intensidad de I+D y las actividades permanentes de I+D, y la probabilidad de llevar a cabo una innovación radical de producto. En este sentido, Leiponen (2006) apunta que la I+D institucionalizada es importante solo para mejorar los servicios existentes, pero no para crear nuevos servicios. En cuanto a los procesos de producción, Cabello-Medina, Carmona y Valle (2006) afirman que los procesos estandarizados están relacionados positivamente con la innovación radical de proceso. De modo que no queda suficientemente clara la relación entre el aprendizaje y la innovación radical de proceso.

Por otra parte, Leiponen (2006) afirma que existe una influencia positiva entre la intensidad de I+D y las actividades permanentes de I+D, y la probabilidad de llevar a cabo una innovación incremental de producto. Asimismo, Cabello-Medina, et al. (2006), indican que los procesos flexibles están relacionados positivamente con la innovación incremental de producto.

Sin embargo, Hayton (2005) no logra mostrar las relaciones señaladas, así como la influencia de la propiedad intelectual sobre las innovaciones de producto y de proceso, como el grado de novedad. Probablemente la ausencia de relación e influencia, se debe a la diferencia de sectores productivos y actividad.

Partiendo de los fundamentos teóricos expuestos, el objetivo de esta investigación es analizar la influencia del aprendizaje organizacional en el proceso de innovación de las NEBT del PCM y LEGATEC, localizadas en la Comunidad de Madrid, España.

\section{METOdOLOGÍA}

La metodología diseñada para llevar a cabo el estudio de casos, y con ello la consecución del objetivo de la investigación, ha sido de tipo cualitativa. Se ha seleccionado esta metodología con base en los fundamentos teóricos, la población objeto de estudio y la escasez de estudios previos 
que analicen la relación propuesta en NEBT. El interrogante que ha guiado el estudio de casos ha sido cómo influye el aprendizaje organizacional en los procesos de innovación de las NEBT.

El estudio se ha diseñado siguiendo las recomendaciones de Eisenhardt (1989) y Yin (1984), es decir, la pregunta de investigación planteada ha sido del tipo "cómo", centrada en el contexto de las siete NEBT del PCM y LEGATEC.

Se aseguró que la recopilación de los datos y su análisis cumplen con los test de validez de construcción, fiabilidad, validez interna y validez externa (Eisenhardt, 1989; Sarabia, 1999; Yin, 1984) de la siguiente manera: utilizando múltiples fuentes de evidencia empírica, un protocolo para el estudio y un caso piloto para refinar los procedimientos de recogida de datos y el contenido de los mismos y haciendo que los informantes fueran objeto de los procedimientos. A su vez, que todas las NEBT compartiesen características comunes para realizar el análisis, utilizando como técnica el denominado pattern-matching, relevante para el análisis de casos explicativos donde los vínculos causales se establecen en forma de narración y para comparar de manera iterativa los acontecimientos comprobados con las proposiciones teóricas, utilizando tablas como técnica específica de tratamiento de datos, para ordenarlos, realizar comparaciones entre los acontecimientos observados y para establecer relaciones entre los datos y las proposiciones derivadas de los fundamentos teóricos.

Como técnica principal de recogida de datos se ha utilizado la entrevista y como fuentes de información complementarias la observación y fuentes secundarias. Las entrevistas fueron focalizadas, individuales y tomaron la forma de conversación informal (Merton, Kiske y Kendal, 1956). Las preguntas fueron extraídas de los fundamentos teóricos ya expuestos, del objetivo de investigación y de la interrogante que ha guiado el estudio. Todas las entrevistas se llevaron a cabo entre noviembre y diciembre de 2008, con un informante por empresa y un promotor o socio fundador y/o director general, con una duración media de treinta minutos cada una. 
Antes de realizar las entrevistas se firmó un acuerdo de confidencialidad para preservar la identidad de las empresas, ya que el estudio forma parte de un proyecto de investigación denominado "Diagnóstico estratégico de activos intangibles" financiado por el Instituto Madrileño de Innovación (IMAFE), aplicado a NEBT en fase de desarrollo (hasta 3 años de edad), que operan en sectores de tecnología alta, media-alta (ingeniería, biotecnología y tecnologías de la información y las comunicaciones), y que presentan gasto de I+D y participan en proyectos de I+D, del PCM y LEGATEC. Además, estas empresas se encuentran inmersas en un proceso social de reflexión y de construcción de la estrategia de innovación. Por esta razón las NEBT han sido identificadas con letras. En la tabla 1 se describe la ficha técnica del estudio.

Tabla 1. Ficha técnica del estudio de casos

\begin{tabular}{|c|c|c|c|c|c|}
\hline NEBT & $\begin{array}{c}\text { SECTOR DE } \\
\text { ACTIVIDAD }\end{array}$ & $\begin{array}{c}\text { No DE EM- } \\
\text { PLEADOS }\end{array}$ & $\begin{array}{c}\text { INCIO DE } \\
\text { ACTIVIDAD }\end{array}$ & UNIDAD FUNCIONAL & $\begin{array}{c}\text { NIVEL DE RES- } \\
\text { PONSABILIDAD }\end{array}$ \\
\hline A & Ingeniería & 26 & 2005 & $\begin{array}{c}\text { Dirección General/Depar- } \\
\text { tamento de Marketing y } \\
\text { Ventas }\end{array}$ & Director General \\
\hline B & Biotecnológica & 9 & 2005 & $\begin{array}{c}\text { Dirección General/Depar- } \\
\text { tamento de Marketing y } \\
\text { Ventas }\end{array}$ & Director General \\
\hline C & Biotecnológica & 7 & 2006 & $\begin{array}{c}\text { Dirección General/Depar- } \\
\text { tamento de Desarrollo de } \\
\text { Negocio }\end{array}$ & Socio Fundador \\
\hline D & Biotecnológica & 14 & 2005 & $\begin{array}{c}\text { Dirección General/Depar- } \\
\text { tamento de I+D }\end{array}$ & Socio Fundador \\
\hline E & Ingeniería & 5 & 2006 & $\begin{array}{c}\text { Dirección General/Depar- } \\
\text { tamento de Marketing y } \\
\text { Ventas }\end{array}$ & Socio Fundador \\
\hline F & $\begin{array}{c}\text { Tecnologías de } \\
\text { Información }\end{array}$ & 8 & 2006 & $\begin{array}{c}\text { Dirección General/Depar- } \\
\text { tamento de Organización } \\
\text { y Servicios de Consultoría }\end{array}$ & Director General \\
\hline G & $\begin{array}{l}\text { Tecnologías de } \\
\text { la Información }\end{array}$ & 15 & 2005 & $\begin{array}{c}\text { Dirección General/Depar- } \\
\text { tamento de Implantación } \\
\text { Tecnológica }\end{array}$ & Socio Fundador \\
\hline
\end{tabular}

Fuente: Elaboración propia 
La estructura de las entrevistas se desarrolló en dos fases. En la primera, se pidió a los informantes que describieran su trabajo en la empresa para después centrarnos en características propias de la misma como sector de actividad, historia, estructura, misión y relaciones con su entorno. La segunda fase se centró en aspectos específicos relacionados con la intensidad de las actividades de I+D y el grado de novedad de la innovación. Para extraer de las conversaciones y discursos los datos de análisis, se grabaron todas las entrevistas y se transcribieron íntegramente después de su realización (Eisenhardt, 1989; Yin, 1984). Como técnicas complementarias para la obtención de datos a tiempo real, se utilizó la observación y las fuentes secundarias. En cuanto a la observación, se tomaron notas de las impresiones sobre las actividades y conversaciones de los miembros de las NEBT. En lo referente a las fuentes secundarias, estas han incluido: páginas web de las NEBT participantes en el estudio; páginas web del PCM y de LEGATEC; la Memoria 2007 y 2008 del PCM; artículos y notas de prensa sobre las empresas analizadas, así como documentos internos e informes confidenciales proporcionados por los entrevistados como informes de capital intelectual y esquemas sobre la estrategia de la empresa.

Tabla 2. Resumen de los datos extraídos de las entrevistas

\begin{tabular}{|lccccccc|}
\hline \multicolumn{1}{|c}{$\begin{array}{c}\text { MEDIDAS DE INPUT } \\
\text { \% de procedimientos documen- } \\
\text { tados de gestión }\end{array}$} & $\mathbf{A}$ & $\mathbf{B}$ & $\mathbf{C}$ & $\mathbf{D}$ & $\mathbf{E}$ & $\mathbf{F}$ & $\mathbf{G}$ \\
\hline $\begin{array}{l}\text { \% de procedimientos documen- } \\
\text { tados de carácter técnico }\end{array}$ & $100 \%$ & $100 \%$ & $100 \%$ & $100 \%$ & $100 \%$ & $100 \%$ & $100 \%$ \\
\hline $\begin{array}{l}\text { № de procesos de desarrollo de } \\
\text { productos }\end{array}$ & 6 & 2 & 5 & 2 & 3 & 2 & 3 \\
\hline № de procesos generales & 3 & & & 3 & $100 \%$ \\
\hline $\begin{array}{l}\text { № de procesos de relación con } \\
\text { cliente/proveedor }\end{array}$ & 6 & 2 & 2 & 1 & 1 & 3 & 3 \\
\hline $\begin{array}{l}\text { \% de Inversión en I+D / Gastos } \\
\text { Totales }\end{array}$ & $90 \%$ & $85 \%$ & $100 \%$ & $75 \%$ & $80 \%$ & $80 \%$ \\
\hline \% de técnicos de I+D (EDP) & $70 \%$ & $78 \%$ & $80 \%$ & $100 \%$ & $70 \%$ & $40 \%$ & $90 \%$ \\
\hline $\begin{array}{l}\% \text { de proyectos de I+D en } \\
\text { desarrollo }\end{array}$ & $100 \%$ & $100 \%$ & $100 \%$ & $100 \%$ & $100 \%$ & $100 \%$ & $90 \%$ \\
\hline
\end{tabular}




\begin{tabular}{|llllllll|}
\hline № de patentes & 2 & 2 & 1 & 1 & 2 & 1 & \\
\hline $\begin{array}{l}\text { № de marcas, nombres comer- } \\
\text { ciales registradas }\end{array}$ & & 1 & 1 & & 3 & 2 & 2 \\
\hline $\begin{array}{l}\text { № de productos que explotan } \\
\text { tales marcas }\end{array}$ & 6 & & & & 2 & & 7 \\
\hline № de licencias obtenidas & & 2 & & & 1 & & \\
\hline $\begin{array}{l}\text { № productos nuevos o en desa- } \\
\text { rrollo en los últimos dos años }\end{array}$ & 14 & 2 & 1 & 1 & 7 & 7 & 9 \\
\hline
\end{tabular}

\section{RESULTADOS}

La tabla 2 muestra los datos obtenidos de las entrevistas de las siete NEBT del PCM y LEGATEC. Estos corresponden a las medidas de input que guiaron las entrevistas y que se han materializado en los resultados que se describen a continuación:

En relación a los protocolos organizacionales se han identificado dos medidas: primero, los procedimientos documentales de gestión, que la totalidad de las NEBT los identifican en un porcentaje igual o superior al $80 \%$, es decir, cinco de las NEBT identifican dichos procedimientos en un $100 \%$, mientras que las dos NEBT restantes lo hacen entre 80 \% (Empresa C) y $90 \%$ (Empresa D), respectivamente. Asimismo, los procedimientos documentales de carácter técnico, fueron identificados al $100 \%$ por todas las empresas.

En cuanto a los procesos, los resultados muestran que los procesos internos relativos al número de procesos de desarrollo de producto, son identificados por las siete NEBT y consideran que tanto la identificación como la codificación de dichos procesos son clave para el funcionamiento de la empresa en su actividad innovadora y su permanencia en el mercado. Sin embargo, los procesos generales no son identificados por todas las empresas; solo las empresas A, E y G identifican tales procesos por los resultados que suponen en sus buenas prácticas, a diferencia de las empresas $B$, $C$, D y F, que no identifican de forma clara estos procesos, generando inconvenientes en la función administrativa debido a la falta de conocimientos 
previos sobre la gestión empresarial. También los procesos generados con la relación cliente-proveedor, fueron identificados por las siete NEBT como un activo de conocimiento necesario para la empresa, ya que permite asegurar mercado para el producto resultante y una constante adaptación al entorno.

En materia de esfuerzo en I+D, las NEBT muestran un porcentaje igual o superior al $75 \%$ de inversión en I+D sobre gastos totales, lo que representa una elevada dotación de recursos en las actividades de I+D. Siguiendo en esta materia, el personal en I+D, fue analizado en términos de porcentaje de técnicos de I+D en dedicación exclusiva (EDP). El resultado muestra un porcentaje igual o superior al $70 \%$, que obedece al alto nivel de especialidad requerida en el desempeño de actividades de I+D.

El desarrollo de proyecto de I+D muestra de forma clara que la totalidad de los proyectos (100 \%) corresponden a la función de I+D en las NEBT.

La propiedad intelectual se materializa en el número de patentes registradas por las empresas; en este caso las empresas tienen entre un registro de patente (empresas C, D y F) y dos registros (empresas A, B y E), a excepción de la empresa $\mathrm{G}$, que no tiene ningún registro de patente. Además, se incluye el número de marcas y nombres comerciales registrados por las NEBT, obteniendo como resultado tres registros la empresa $E$, dos registros las empresas $E$ y $F$, un registro las empresas $B$ y $C$, y ningún registro las empresas A y D. También se identifica el número de productos que explotan tales marcas, siendo seis productos (empresa A), dos productos (empresa E) y siete productos (empresa G). Las empresas B, C, D, y F, no tienen registros de productos que exploten sus marcas. En cuanto al número de licencias obtenidas, fueron registradas dos licencias por la empresa B y una por la empresa E. Las cinco NEBT restantes (empresas A, C, D, F y G), no presentan registros de licencias.

Finalmente, se identifica el número de productos nuevos o en desarrollo en los últimos años como resultado de la innovación. A este respecto, todas las empresas han obtenido resultados satisfactorios como conse- 
cuencia de los proyectos de I+D que realizan y las alianzas con los proveedores. Los resultados muestran catorce productos nuevos o en desarrollo (empresa A), dos (empresa B), uno (empresas C y D), siete (empresas E y F), y nueve (empresa G).

De los resultados del estudio se corrobora la influencia del aprendizaje organizacional como proceso en el desarrollo de innovaciones, a partir de la relación directa que existe entre el conocimiento y el aprendizaje materializado en el esfuerzo en I+D que realizan las NEBT, es decir, el gasto en $I+D$, la participación en proyectos de I+D, y personal de dedicación exclusiva en la I+D. En cuanto a la propiedad intelectual, al igual que en la revisión de los estudios empíricos no queda clara la relación positiva que esta puede tener con el resultado innovador, aunque sí está vinculada al proceso de aprendizaje, ya que son resultados de la acumulación de experiencia y de ampliación de la base de conocimiento de las empresas. Dicho esto, la influencia del aprendizaje organizacional está sujeta a la ambigüedad causal de sus implicaciones en el rendimiento, especialmente relevante en entornos sometidos a cambios rápidos.

\section{CONSIDERACIONES FINALES}

De los resultados obtenidos en el estudio se desprende que el aprendizaje organizacional influye de forma positiva en el proceso de innovación. Concretamente, se reconocen las NEBT analizadas como sistemas de aprendizaje capaces de reducir la brecha entre los conocimientos existentes en la organización y los que son necesarios para responder eficientemente a las exigencias percibidas del entorno. Todo ello dependerá de la interpretación que la NEBT haga de la realidad y de su potencial de respuesta por medio del aprendizaje. En otras palabras, la capacidad de aprendizaje de una NEBT es resultante de un ciclo continuo de articulación y codificación de conocimientos que se relacionan y se refuerzan mutuamente, capacitando al sistema para crear, sostener y generalizar conocimientos con impacto, es decir, innovación. 
GESTIÓN DEL CONOCIMIENTO Y CAPACIDAD DE INNOVACIÓN

Modelos, Sistemas y Aplicaciones

Desde esta óptica cabe destacar la importancia del esfuerzo en I+D como negocio que caracteriza a las NEBT. La I+D como negocio representa un identificativo estratégico fundamental ya que se conjuga con las siguientes consideraciones:

- La generación y desarrollo del conocimiento son los procesos clave.

- El esfuerzo en I+D debe ser continuo.

- La oferta siempre busca ser vanguardista.

- Comúnmente, la llegada al mercado genera un gap entre el marco técnico y el comercial. Un ejemplo son los activos de propiedad intelectual y su escasa explotación comercial, tal y como se muestra en la revisión de los estudios empíricos y el estudio de casos.

La enunciación de estos aspectos permite argumentar que el aprendizaje en las NEBT favorece, como ya se ha dicho, la acumulación de experiencia, la articulación de conocimiento y la codificación de conocimiento, que se traduce en rutinas organizacionales que implican la ejecución de procedimientos que generan rendimientos y cambios deseables en el desarrollo de capacidades.

Sin duda, un elemento diferencial en la orientación hacia la I+D como negocio está en reconocer la complejidad y amplitud de conocimientos que son necesarios para innovar, y, por tanto, la idoneidad o imperativo alrededor de la construcción de un marco relacional potente donde los flujos de información y conocimiento sean de calidad (Raju, 2006).

Conforme a los resultados obtenidos, se pretende ofrecer algunas guías de acción, en general, dirigidas a mantener a las NEBT en un estado de mejora continuada en el desarrollo de su capacidad de aprendizaje y, por ende, de su innovación. Estas sugerencias, como el desarrollo teórico, están sujetas a revisión, por tratarse de empresas en constantes cambios.

Es más, el esfuerzo organizacional, en su conjunto, debe ser estructurado contando con un esquema empresarial dotado de funciones y responsabilidades definidas, procesos formalizados, protección de los resultados, etc., asegurando las dinámicas de gestión en todas las áreas funcionales. 
La identificación de experiencias empresariales o emprendedoras previas, además de la existencia de perfiles especializados para la gestión, favorece en las NEBT la existencia de una estructura organizacional definida, la formalización de procesos y el esfuerzo en I+D. Todos ellos, aspectos que permiten dilucidar el grado de madurez de la empresa, y, por ende, la superación del voluntarismo y posible caos de las primeras fases. Es más, se logra diferenciar si la orientación hacia el I+D como negocio es real o se quiere hacer un negocio con un resultado concreto de la I+D acumulada durante etapas pre-empresa.

Siempre conviene analizar las características del mercado que se pretende atacar y el soporte derivado del marco de contactos y alianzas. Todo ello se complementa con el ámbito social en el que se valoran activos tan significativos hoy en día como la notoriedad, imagen y reputación, que no fueron incluidos en las medidas de input, pero sí fueron analizados en las fuentes de información complementarias, por tratarse de activos necesarios en el desarrollo del negocio.

Los resultados del estudio han favorecido la articulación de prioridades en la gestión para hacerla más completa, eficiente y profesional, ajustando las variables de la I+D, en aquellas que el negocio demanda. Además, los resultados se presentan como una herramienta de comunicación importante dado que aflora una realidad de valor que generalmente se encuentra escasamente definida, y que en procesos donde se pretende la valoración por terceros resulta un requerimiento crítico para mejorar el atractivo de la organización.

Sin duda, la visualización de aspectos humanos (técnicos en I+D), estructurales (esfuerzo en I+D y protocolo organizacional) y relacionales (proyectos de $I+D$, relación cliente-proveedor y propiedad intelectual), orientan el valor del estudio hacia la creación de una verdadera NEBT, con visos de sostenibilidad lo que alude a su contribución en los estadios de consolidación del proyecto empresarial.

Desde esta perspectiva, el conjunto de las NEBT se configura como un 
colectivo especial. Los códigos de conducta, la percepción del tiempo y los perfiles que la conforman, son aspectos especialmente críticos a la hora de realizar el tratamiento de datos, y, más aún, en el momento de construir recomendaciones.

\section{REFERENCIAS BIBLIOGRÁFICAS}

Acosta, J. C. (2010). Creación y desarrollo de capacidades tecnológicas: Un modelo de análisis basado en el enfoque de conocimiento. (Tesis doctoral). Universidad Autónoma de Madrid. Madrid, España.

Adams, R., Bessant, J. \& Phelps, R. (2006). Innovation Management Measurement: A Review. International Journal of Management Reviews, 8, 21-47.

Adner, R. (2002). When are Technologies Disruptive? A Demand-Based View of the Emergence of Competition. Strategic Management Journal, 23, 667-688.

Alle, V. (1997). The Knowledge Evolution. Expanding Organizational Intelligence. Boston: Buttterworth-Heinemann.

Andreu, R. \& Ciborra, C. (1994). Information Systems for the Learning Organization. Speech delivered Technology and Organizational Change. Nijenrode University.

Andreu, R. \& Ciborra, C. (1995). The Role of IT in Creating an Effective Knowledge Base for the Learning Organization, EFMD Forum, 1.

Andreu, R. \& Ciborra, C. (1996). Core Capabilities and Information Technology: An Organisational Learning Approach. In B. Moingeon \& A. Edmondson. Organizational and Competive Advantage. Londres: Sage.

Appelbaum, S. H. \& Reichart, W. (1997). How to Measure an Organization's Learning Ability: A Learning Orientation (part I). Journal of Workplace Learning, 9(7), 225-239.

Argyris, C. \& Schön, D. (1978). Organizational Learning: A Theory of Action Perspective. Reading, M.A.: Addison Wesley. 
Bahlmann, T. (1990). The Learning Organization in a Turbulent Environment. European Journal of Management Research, 9(4), 167-182.

Benavides, C. A. (1998). Tecnología, Innovación y Empresa. Madrid: Pirámide.

Beveridge, M., Gear, A. E. \& Minkes, A. L. (1997). Organizational Learning and Strategic Decision Support. The Learning Organization, 4(5), 217-227.

Cabello-Medina, C., Carmona-Lavado, A. \& Valle-Cabrera, R. (2006). Identifying the Variables Associated with Types of Innovation, Radical or Incremental: Strategic Flexibility, Organisation and Context. International Journal of Technology Management, 35, 80-106.

Cangelosi, V. E. \& Dill, W. R. (1965). Organizational Learning Observations: Toward a Theory. Administrative Science Quarterly, 10, 175-203.

Cohen, W. \& Walsh, J. (2000). R\&D spillovers, appropiability and R\&D intensity: a survey based approach. Mimeo, Carnegie Mellon University.

Cohen, W., Goto, A., Nagata, A., Nelson, R. \& Walsh, J. (2002). R\&D spillovers, patents and the incentives to innovate in Japan and the United States. Research Policy, 31 (8/9), 1349-1367.

Cohen, W. M. \& Levinthal, D. A. (1990). Absorptive Capacity: a New Perspective on Learning and Innovation. Administrative Science Quarterly, 35, 128-152.

Crossan, M. M., Lane, H. W. \& White, R. E. (1999). An Organizational Learning Framework: from Intuition to Institution. Academy of Management Review, 24(3), 522-537.

Cyert, R. M. \& March, J. G. (1963). A Behavioural Theory of the Firm. Englewood Cliffs, NJ: Prentice-Hall.

DiBella, A. J. \& Nevis, E. (1998). How Organizations Learn. San Francisco: Jossey Bass.

DiBella, A.J., Nevis, E.C. \& Gould, J.M. (1996). Understanding Organizational Learning Capability. Journal of Management Studies, 33(3), 361-379. 
Dodgson, M. (1993). Organizational Learning: A Review of Some Literatures. Organizations Studies, 14, 375-394.

Duncan, R. \& Weiss, A. (1979). Organizational Learning: Implications for Organizational Design. Research in Organizational Behaviour, 1, 75-123.

Eisenhardt, K. M. (1989). Building theories from case study research. Academy of Management Review, 14, 532-550.

Fiol, C. M. \& Lyles, M. A. (1985). Organizational Learning. Academy of Management Review, 10(4), 803-813.

Gallego, A. y Casanueva, C. (2007). El Peso de la Cooperación en la Innovación de la Empresa Industrial Española. XVII Congreso Nacional de ACEDE. Comunicación Oral, Sevilla.

Garratt, B. (1987). The Learning Organization: and the need for directors who think. Cambridge: Gower.

Garratt, B. (1990). Creating a Learning Organization: a guide to Leadershipnd, Learning and Development. Londres: Director Book.

Garratt, B. (1992). Continuar Aprendiendo. Formación y Empresa, 33, 6-7.

Garvin, G. A. (1993). Building a Learning Organization. Harvard Business Review, 71, 78-91.

Hamel, G. \& Prahalad, C. (1993). Strategic as Stretch a Leverage. Harvard Business Review, 71(2), 75-84.

Hayton, J. C. (2005). Competing in the New Economy: The Effect of Intellectual Capital on Corporate Entrepreneurship in High-Technology New Ventures. $R$ \& D Management, 35, 137-155.

Hedberg, B. (1981). How Organizations Learn and Unlean. In, P. Nystrom, \& W. Stabuck. Handbook of Organizational Desing, (pp.3-27). New York: Oxford University Press.

Hegde, D. \& Shapira, P. (2007). Knowledge, Technology Trajectories, and Innovation in a Developing Country Context: Evidence from a Survey of Malaysian Firms. International Journal of Technology Management, 40, 349-370. 
Huber, G. (1991). Organizational Learning: The Contributing Processes and the Literatures. Organization Science, 2, 88-115.

Huergo, E. (2006). The Role of Technological Management as a Source of Innovation: Evidence from Spanish Manufacturing Firms. Research Policy, 35, 1377-1388.

Kim, D. (1993). The Link Between Individual and Organizational Learning. Sloan Management Review, fall, 37-50.

Kogut, B. \& Zander, U. (1992). Knowledge of the Firm, Combinative Capabilities, and the Replication of Technology. Organization Science, 3(3), 383-397.

Landier, H. (1992). Hacia la empresa inteligente: Guía para la gestión del cambio. Barcelona: Ediciones Deusto, S.A.

Leiponen, A. (2006). Managing Knowledge for Innovation: The Case of Business-to-Business Services. Journal of Product Innovation Management, 23, 238-258.

Leonard Barton, D. (1993). La fábrica como laboratorio de aprendizaje. Harvard Deusto Business Review, 58, 46-61.

Leonard Barton, D. (1995). Wellsprings of Knowledege: Building and Sustaining the Sources of Innovation. Boston: Harvard Business School Press.

Levitt, B. \& March, J. (1988). Organizational Learning. American Review of Sociology, 14, 24-39.

López Mielgo, N., Montes Peón, J., Vásquez Ordas, C. \& Prieto Rodríguez, J. (2004). Innovación y competitividad: implicaciones para la gestión de la innovación. Revista Madrid, 24.

March, J. \& Olsen, J. (1976). Organizational learning and the ambiguity of the past. In J. March, \& J. Olsen, (eds.). Ambiguity and choice in organizations (pp.54-68). Bergen, Noruega: Universitetsforlaget.

Marengo, L. (1991). Knowledge, Coordination and Learning in an Adaptive Model of Firm (Tesis doctoral). Sussex University, Sussex.

McEvily, S. K. \& Chakravarthy, B. (2002). The Persistence of Knowledge-Ba- 
sed Advantage: An Empirical Test for Product Performance and Technological Knowledge. Strategic Management Journal, 23, 285-305.

Merton. R. K., Kiske, M. \& Kendal, P. L. (1956). The Focused Interview. Glencoe, III: Free Press.

Muñoz-Seca, B. \& Riverola, J. (1997). Gestión del Conocimiento. Folio. Barcelona.

Nevis, E., Dibella, A. \& Gould, J. (1995). Understanding organizations as learning systems. Sloan Management Review, Invierno, 73-85.

Nonaka, I. (1999). The Dynamics of Knowledge Creation. In R. Ruggles \& D. Holthouse (eds.). The Knowledge Advantage. Capstone (pp.63-88).

Nonaka, I. \& Ichijo, K. (1997). Creating Knowledge in the Process Organization. In J. P. Walsh \& J. S. Huff (1997). Advances in Strategic Management, 14, 45-52.

Nonaka, I. \& Takeuchi, H. (1995). The knowledge-creating company. Oxford: Ed. Oxford University Press.

Nonaka, I., Reinmoeller, P. \& Senoo, D. (1998). Management Focus; the "ART" of Knowledge: Systems to Capitalize on Market Knowledge. European Management Journal, 16(6), 673-684.

Polanyi, M. (1962). Personal Knowledge. Chicago: University of Chicago Press.

Prajogo, D. I. \& Ahmed, P. K. (2006). Relationships between Innovation Stimulus, Innovation Capacity, and Innovation Performance. $R$ \& D Management, 36, 499-515.

Probst, G. \& Büchel, B. (1995). La Practique de l'Entreprise Apprenante. Les Éditions d'Organosation. París: Éditions d'Organosation.

Raju, P. U. (2006). Creating business value through people. International Journal of Learning and Intellectual Capital, 3(4), 357-366.

Rant, M. (2001). The Role of Organization in Knowledge Management: The Empirical Study of the Organization as the Focal Knowledge Creation and Sharing Mechanism. Second European Conference on Knowledge Management. Bled, Slovenia. 
Redding, J. y Catalanello, R. (1994). Strategic Readiness. San Francisco: Jossey-Bass.

Revilla, E. (1998). De la organización que aprende hacia la dirección del conocimiento. VIII Congreso Nacional de ACEDE. Las Palmas de Gran Canaria.

Rogers, E. (1983). The Diffusion of Innovation. Nueva York: Free Press.

Sánchez, R. \& Heene, A. (2000). A competence perspective on strategic learning and knowledge management. In R. Cross, \& S. Israelit, (eds.). Strategic Learning in a Knowledge Economy: Individual, Collective, and Organizational Learning Process (pp.23-35). Oxford: Butterworth-Heinemann.

Sarabia, F. J. (1999). Metodología para la investigación en marketing y dirección de empresas. Madrid: Ed. Pirámide.

Senge, P. (1990). The Fifth Discipline. New York: Doubleday.

Shrivastava, P. (1983). A tipology of organizational learning systems. Journal of Management Science, 20, 2-24.

Swieringa, J. \& Wierdsma, A. (1992). Becoming a learning organization. México: Addison Wesley Publishing Company, Inc.

Teece, D., Pisano, G. \& Shuen, A. (1997). Dynamic Capabilities and Strategic Management. Strategic Management Journal, 18, 509-533.

Van der Krogt, F. J. (1998). Learning Network Theory: The Tension Between Learning Systemas and Work Systemas in Organizations. Human Resource Development Quarterly, 9(2), 156-176.

Vera, D. \& Crossan, M. (2000). Organizational Learning, Knowledge Management, and Intellectual Capital: An Integrative Conceptual Model. Working Paper.

Walsh, J. \& Ungson, G. (1991). Organizational Memory. Academy of Management Review, 16(1), 57-91.

Watkins, K. \& Marsick, V. (1993). Sculpting the learning organization. San Francisco: Jossey-Bass. 
Weick, K. E. (1991). The Nontraditional Quality of Organizational Learning. Organization Science, 2(1), 116-123.

West, M. A. \& Farr, J. L. (1990). Innovations and creativity at work: Psychological and organizational strategies. Chichester: Wiley.

Yam, R. C. M., Cheng, J., Fai, K. \& Tang, E. P. Y. (2004). An Audit of Technological Innovation Capabilities in Chinese Firms: Some Empirical Findings in Beijing, China. Research Policy, 33, 1123-1140.

Yin, R. K. (1984). Case Study Research: Design and Methods. California: Ed, Sage Publications.

Zack, M. H. (1999). Developing a Knowledge Strategy. California Management Review, 41(3), 125-145. proceso de innovación. Una aplicación en nuevas empresas de base tecnológica. En K. Barrios Hernández, E. Olivero Vega, J. C. Acosta Prado, I. Meñaca Guerrero, A. M. Cazallo Antúnez, H. J. Medina Carrascal, ... O. Hernández Ariza, Gestión del conocimiento y capacidad de innovación. Modelos, Sistemas y Aplicaciones (pp.315-338). Barranquilla: Universidad Simón Bolívar. 